\title{
Inverse rule of mixtures at the nanoscale: prediction of elastic properties of cellulose nanofibrils
}

\author{
Ali Khodayari ${ }^{\mathrm{a}, *}$, Ulrich Hirn ${ }^{\mathrm{b}}$, Aart W. Van Vuure ${ }^{\mathrm{a}}$, David Seveno ${ }^{\mathrm{a}}$ \\ ${ }^{a}$ Department of Materials Engineering, KU Leuven, Leuven, Belgium \\ ${ }^{b}$ Institute of Bioproducts and Paper Technology, TU Graz, Graz, Austria
}

\begin{abstract}
A way to model properties of nanomaterials consists of applying well-established approaches based on a macroscopic view of matter, therefore ignoring any peculiar features due to the smallness of these materials. In this work, we inspect the capability of a simple micromechanical model, the inverse rule of mixtures, to predict the elastic modulus of multi-component composites, e.g. cellulose nano fibrils, when load is perpendicular to the stratified structure, by implementing molecular dynamics simulations. The results show that the inverse rule of mixtures can predict the tensile modulus of the modelled cellulose nano fibrils in the fibril directions. These findings can be further generalized in computing the mechanical properties of multi-phase nanocomposites, in case the constituents are loaded in series, as well as, to approximate the degree of crystallinity of single cellulose nano fibrils. Keywords: Cellulose, Nano-structures, Elasticity, Computational modelling
\end{abstract}

\section{Introduction}

Describing the laws of nature has for centuries been based on a continuum view of matter leading, to list a very few, to the very well-known Newton's, Hooke's, or Navier-Stoke's equations. On the other hand, the emergence of quantum mechanics and nanotechnology during the 20th century gave rise to other fundamentally different views. If it is established that quantum effects cannot be described by a classical mechanical approach, the length scale at which a continuum description of matter becomes valid remains subject of a vivid debate. Indeed, at the nanoscale, peculiar behaviour like frictionless flow in carbon nanotubes [1], very high heat conduction in graphene [2, 3], and the existence of a dynamic contact angle [4] have been observed and characterized based on a discrete description of matter. In parallel and at the same scale, well-established laws and phenomena derived from a

\footnotetext{
* Corresponding author

Email address: ali.khodayari@kuleuven.be (Ali Khodayari)
} 
macroscopic, i.e. continuum approach, have also been validated like Young's equation [5], the presence of a Poiseuille flow in a nano-capillary [6], the Wilhelmy equation [7, 8, Amontons' law [9], Kirchhoff's superposition law in carbon atomic wires [10, and the Bruggeman approximation for thermal properties [1]. On the other hand, certain universal laws such as Fourier's law are claimed to be invalid for the low dimensional structures [12, while the thermal conductivity of small-scale systems has been a topic of discussion for years [13, 14]. Mechanical properties of nano-composites are also described by popular composite theories at the macroscale [15. How the inverse rule of mixtures (IROM) is applicable to define the wear mechanism of nano-composites has been evaluated [16]. The same rule is shown to be valid to define the tensile modulus of ternary nano-composites, with some modifications [17]. However, the applicability of the rule was evaluated for nano-composites in the form of a matrix with reinforcing phase. Attempts to introduce modifications to the micromechanical models predicting the tensile properties of nano-composites have also been made [18. In this work, we investigate a novel application of the IROM, to describe the composite elastic modulus at nanoscale. The rule is valid for configurations where the loading direction is perpendicular to a stratified composite structure of two or more phases. It gives an exact solution in those cases where there is a perfect series connection of the constituents.

Basically, composites consisting of two or more phases can be divided into two categories. Mixtures of a reinforcing material and matrix, where the applied load would not equally be distributed in the two phases. This category is the case for the majority of the nano-reinforced composites. The second category however is referring to nano-composites where components are placed in series, in the direction of the applied load. The latter category will experience an isostress situation, if the Poisson effect is not present or can be neglected. The strain in each section of the composite in this case will be a function of the elastic modulus of the individual components. Such an exemplary structure is a cellulose nanofibril (CNF), a nano-composite of crystalline and dislocated cellulose, under longitudinal load.

For a two phase composite (c and d), stretching of two springs connected in series can be expressed as the summation of elongations in the two springs

$$
\Delta l_{t o t}=\Delta l_{c}+\Delta l_{d}
$$

where $\Delta l_{\text {tot }}$ is the total elongation and $\Delta l_{c}$ and $\Delta l_{d}$ are the elongations in the two individual 
springs. Having that $\Delta l=\epsilon l$, being $\epsilon$ the strain and $l[\mathrm{~m}]$ the length, we get

$$
\epsilon_{t o t} l_{t o t}=\epsilon_{c} l_{c}+\epsilon_{d} l_{d}
$$

substituting Hooke's law $\sigma=E \epsilon$, having that $\sigma\left[\frac{N}{m^{2}}\right]$ and $E\left[\frac{N}{m^{2}}\right]$ are the stress and the elastic modulus, respectively, we obtain

$$
\frac{\sigma_{t o t} l_{t o t}}{E_{t o t}}=\frac{\sigma_{c} l_{c}}{E_{c}}+\frac{\sigma_{d} l_{d}}{E_{d}}
$$

Assuming now that the forces in each segment are equal (series of springs) and the cross sections are equal (i.e. neglecting density differences) we can justify the isostress assumption $\sigma_{t o t}=\sigma_{c}=\sigma_{d}$, and cancelling these terms leads to

$$
\frac{l_{t o t}}{E_{t o t}}=\frac{l_{c}}{E_{c}}+\frac{l_{d}}{E_{d}}
$$

Again considering a constant cross section $A$ (i.e. neglecting density differences) we find the volume in each element $V=l A$ and thus the IROM:

$$
\frac{1}{E_{t o t}}=\frac{v_{c}}{E_{c}}+\frac{v_{d}}{E_{d}}
$$

Where $E_{t o t}$ is the equivalent elastic modulus, $E_{c}$ and $E_{d}$ are the elastic moduli, and $v_{c}$ and $v_{d}$ are the volume fractions of the two segments. Following the ideas developed above it is straightforward to see that the IROM can also describe the elastic modulus of multi-phase composites with $\mathrm{n}$ components loaded in series, having volumetric ratios of $v_{1}, v_{2}, \ldots, v_{n}$, and corresponding elastic moduli of $E_{1}, E_{2}$, $\ldots, E_{n}$, as follows

$$
\frac{1}{E_{t o t}}=\frac{v_{1}}{E_{1}}+\frac{v_{2}}{E_{2}}+\ldots+\frac{v_{n}}{E_{n}}
$$

CNFs are components of the plant cell wall, consisting of two phases of crystalline and dislocated regions. While crystalline segments are highly ordered, showing high values of elastic moduli, dislocated regions, having a lower number of hydrogen bonds, intrinsically exhibit an elastic modulus of almost half of that of the crystals [19]. Although there is controversy in literature, dislocated segments of the $\mathrm{CNF}$ are believed to be located in series with the cellulose crystals, impacting the overall mechanical properties 20. The structure of the CNFs can hence be schematized and modelled as springs loaded 


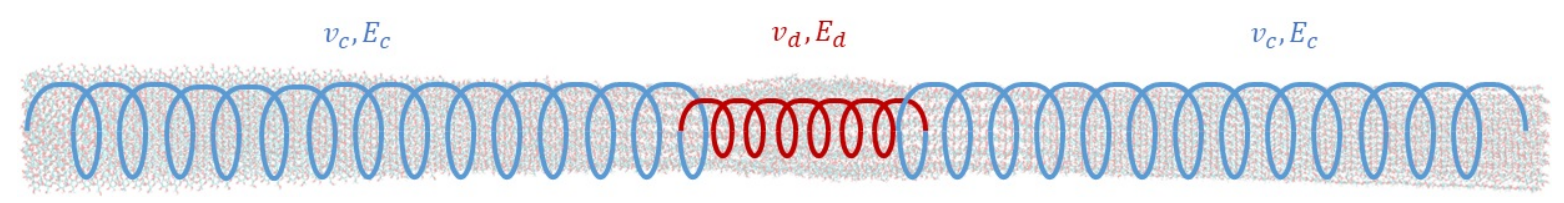

Figure 1: The schematic of a CNF, and its equivalent spring structure. The blue and red sections represent the crystalline and dislocated segments, respectively. Consequently, $E_{c}$ and $E_{d}$ represent the elastic moduli, and $v_{c}$ and $v_{d}$ denote the volume fractions of the crystalline and dislocated segments, respectively.

in series, as illustrated in Fig 1 .

In this paper, we compare the simple relationship of the IROM to predict the longitudinal Young's modulus, with molecular-dynamics simulations used to calculate the same property, for a composite cellulose nanofibril made of crystalline and dislocated segments. The aim is thus to verify the validity of IROM, being a continuum model, when applied at the nanoscale. It is shown how the tensile properties of the CNFs are affected by the presence of dislocated segments. The results could potentially shed light on defining the degree of polymerization of CNFs, and the content of the dislocated segments by reverse engineering the tensile modulus of the fibrils.

\section{Methods}

\subsection{Molecular dynamics simulations}

Molecular Dynamics simulations are performed by the Groningen Machine for Chemical Simulations (GROMACS), 2018.7 version [21. The GLYCAM06 force field specified for modelling carbohydrates is used [22]. The parameter sets are then converted to GROMACS format using the AnteChamber Python Parser interface (ACPYPE) python code 23] from the source Amber package 24]. A quadratic polynomial is used to model the bonded interactions including stretching and angle potential, whereas torsional potentials are modelled with a three-term Fourier expansion series. Van der Waals interactions are treated with a $6-12^{\text {th }}$ power Lennard-Jones potential, and Coulombic potential is used to treat the electrostatic interactions. The steepest decent algorithm is employed to minimize the structure's energies. Newton's equations of motion are solved with a leap-frog algorithm in the canonical ensemble (constant Number of atoms, constant Volume, and constant Temperature), and production runs. Bonded hydrogens are constrained through the LINCS algorithm. Neighbour searching is performed with a Verlet scheme, having a cut-off for both the van der Waals and short-range electrostatic interactions of $1.2 \mathrm{~nm}$. Electrostatics are treated with particle-mesh Ewald (PME). A velocity rescaling thermostat is used to control the temperature of different sections of the fibrils [25]. The time-step 
of the simulations is set to $2 \mathrm{fs}$. Pulling is performed by applying a force at specific positions on the fibrils.

\subsection{Models}

The $6 \times 6$ crystal structure is modelled in Materials Studio [26] using the crystallographic data for I $\beta$ cellulose reported in the literature [27. The simulated 36-chain structure with a degree of polymerization of 119 (DP 119) has a length of $63.5 \mathrm{~nm}$. To model the dislocations in the middle of the fibrils, a previously reported procedure was applied [19]. The specified residues are tempered to 750,900 , and $1020 \mathrm{~K}$ for $1 \mathrm{~ns}$ at each step. The resulting structure is then quenched down to $300 \mathrm{~K}$ for another $1 \mathrm{~ns}$. The cross-sectional area of the fibrils is computed by multiplying the distances between the side chains, considering the cross-section to be a rectangle. The chain distances are multiplied by correction factors to reach the correct approximate cross-section of $10.5 \mathrm{~nm}^{2}$, as the true chain distance is slightly more than the center of mass to center of mass of the chains. The final area is the average of 8 computed cross-sections over the length of the fibrils.

The IROM can be investigated in models with different sizes of dislocations providing different volume fractions. Fig $2 \mathrm{a}$ shows the $63.5 \mathrm{~nm} 6 \times 6 \mathrm{I} \beta$ cellulose nano crystal $(\mathrm{CNC})$, used to accommodate dislocated segments in the middle. To vary volume fractions, dislocated segments with different sizes were modelled, but at higher levels of dislocated materials, fibrils were bent at the dislocated segment making it difficult to measure the elastic modulus. This is because the pulling force applied at the two ends of the fibril is used to simultaneously unbend and stretch it (see Fig 2c). The most stable model was then chosen to investigate the IROM, having 10 glucose units dislocated in the middle, as depicted in Fig 2b,

To mimic various volume fractions of the dislocated segment, forces were applied at different positions on the CNF, e.g. if forces are applied at the two ends of the dislocated segment, a model containing $100 \%$ dislocation is achieved. If the force is applied at two positions being 50 glucose units apart, this gives $20 \%$ of dislocation volume fraction and so forth (see Fig 3). The same procedure was applied to obtain 13 points, including 13 different elastic moduli and dislocated segment content.

\section{Results and discussion}

Results of the computed elastic moduli through the simulations and by IROM are depicted in Fig.4. To calculate the elastic moduli by the IROM, the elastic modulus of the crystalline and dislocated 


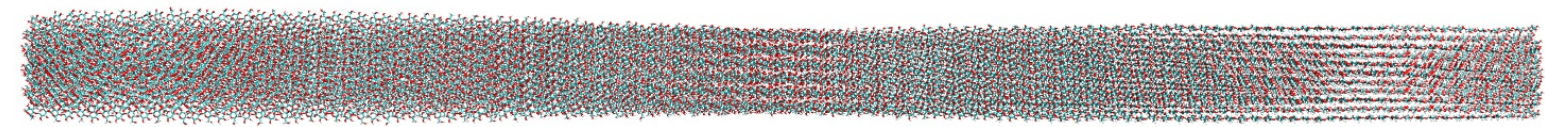

(a)

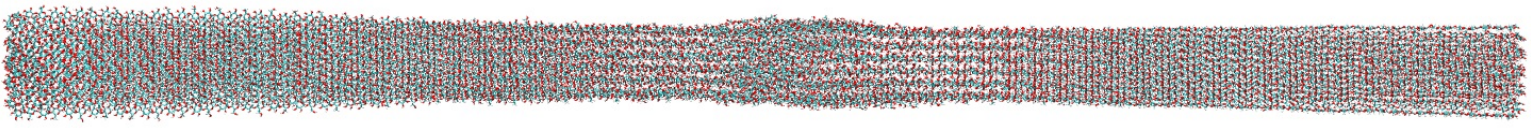

(b)

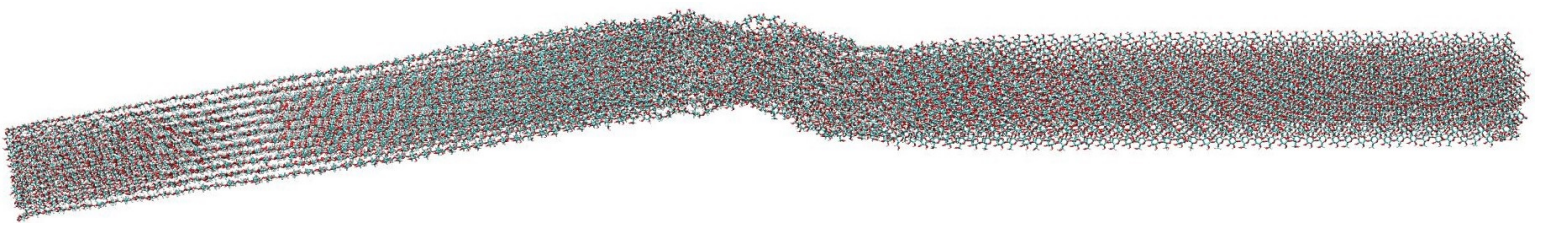

(c)

Figure 2: (a) The fully crystalline model, (b) CNF including 10 glucose units of dislocated segments in the middle, (c) CNF including 25 glucose units of dislocated segments in the middle. The last model bent during the production, making it difficult to measure the elastic modulus.

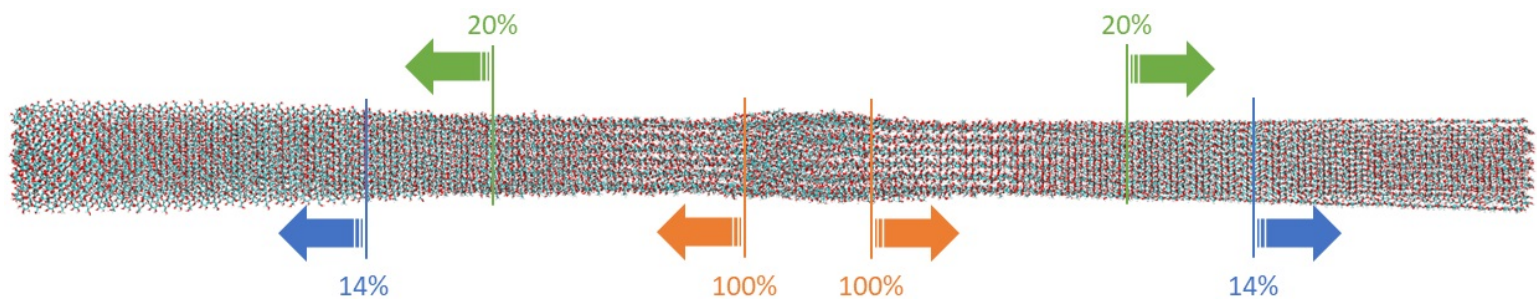

Figure 3: Points at which the force is applied can be varied to change the volume fraction of the dislocated segments. For example applying the forces at the blue sections is equal to have a CNF with $14 \%$ of dislocated segment, whereas the two ends are loose and play no role. 


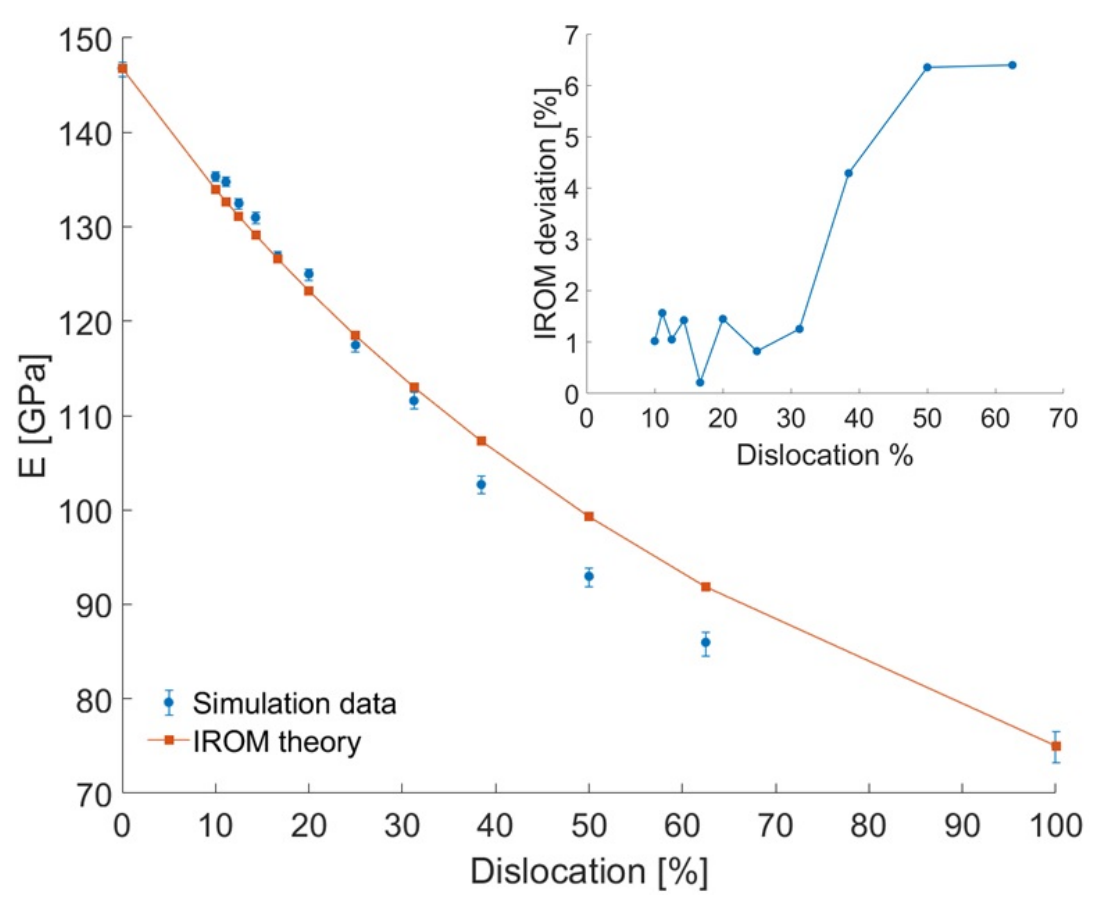

Figure 4: Results of the elastic moduli versus the percentage of the dislocations in the CNF. The blue circles represent the simulation results, whereas the red squares show the IROM. Results of IROM show very good agreement with the simulations. The inset shows the deviation error of the simulation results from that of the IROM.

segments were computed in the simulations and are $146.7 \mathrm{GPa}$ and $74.8 \mathrm{GPa}$, respectively (for $5 \%$ of strain). As can be seen, there is a complete agreement between the theoretical and numerical results up to $32 \%$ of dislocation content. Errors start to increase when the percentage of crystalline units becomes so small that the discretization leads to deviations from the continuous expression of the IROM. This inherently increases the errors in cross-sectional area calculation, as the segments where the dislocation is present are not as ordered as the crystalline sections. Thus, the increasing error for the last three points is associated with the cross-sectional area calculation. Moreover, including more points in between $62 \%$ and $100 \%$ is simulation-wise not possible. This is because, considering 10 glucose units of dislocation, including only three glucose units of crystalline segments at the two ends of the dislocated segment already drops the dislocation percentage to $60 \%$.

\section{Conclusions}

In this work, we investigated the applicability of the inverse rule of mixtures to predict the modulus of elasticity for nano-composites, by demonstrating it for cellulose nanofibrils. A model including a $5.3 \mathrm{~nm}$ dislocated segment in the middle of a $63.5 \mathrm{~nm}$ cellulose fibril was simulated, while forces were applied at different positions along the length of the fibril, mimicking different volume fractions. 
The elastic moduli versus the percentage of dislocated segments in the fibril was then compared with that of the inverse rule of mixtures. Results completely confirm that the elastic modulus of cellulose nanofibrils can be estimated by the IROM, knowing the volumetric fraction of the dislocated segments in the medium. As a side application, the results can be utilized in future studies, where experimental testing of single CNFs is possible. The inverse rule of mixtures can be used to estimate the crystallinity index of the fibrils, in case data on the elastic modulus of individual fibrils is available. On a more general level we have validated the IROM for a two-phase composite where the phases are mechanically loaded in series (i.e. a 2-phase stratified composite where the loading direction is perpendicular to its layers). This work can bridge the nanoscale to higher scales by providing a formula for the mechanical properties of certain multicomponent composites, which are often difficult to test directly.

\section{acknowledgement}

The authors are grateful to Prof. Wim Thielemans and Prof. Eric Nies for their invaluable discussions. The computational resources and services used in this work were provided by the VSC (Flemish Supercomputer Center), funded by the Research Foundation - Flanders (FWO) and the Flemish Government - department EWI. This project has received funding from the European Union's Horizon 2020 research and innovation programme under the Marie Skłodowska-Curie [grant agreement No 764713], project FibreNet.

\section{Competing interests}

Declarations of interest: none.

\section{References}

[1] K. Falk, F. Sedlmeier, L. Joly, R. R. Netz, L. Bocquet, Molecular origin of fast water transport in carbon nanotube membranes: Superlubricity versus curvature dependent friction, Nano Letters 10 (10) (2010) 4067-4073. doi:10.1021/nl1021046.

[2] A. A. Balandin, S. Ghosh, W. Bao, I. Calizo, D. Teweldebrhan, F. Miao, C. N. Lau, Superior thermal conductivity of single-layer graphene, Nano Letters 8 (3) (2008) 902-907. doi:10.1021/ n10731872. 
[3] A. A. Balandin, Thermal properties of graphene and nanostructured carbon materials, Nature Materials 10 (8) (2011) 569-581. doi:10.1038/nmat3064.

[4] A. V. Lukyanov, A. E. Likhtman, Dynamic Contact Angle at the Nanoscale: A Unified View, ACS Nano 10 (6) (2016) 6045-6053. doi:10.1021/acsnano.6b01630.

[5] J. C. Fernandez-Toledano, T. D. Blake, P. Lambert, J. De Coninck, On the cohesion of fluids and their adhesion to solids: Young's equation at the atomic scale, Advances in Colloid and Interface Science 245 (2017) 102-107. doi:10.1016/j.cis.2017.03.006.

[6] G. Martic, F. Gentner, D. Seveno, D. Coulon, J. De Coninck, T. D. Blake, A molecular dynamics simulation of capillary imbibition, Langmuir 18 (21) (2002) 7971-7976. doi:10.1021/la020068n.

[7] D. Seveno, T. D. Blake, J. De Coninck, Young's equation at the nanoscale, Physical Review Letters 111 (9) (2013) 1-4. doi:10.1103/PhysRevLett.111.096101

[8] C. Mortagne, K. Lippera, P. Tordjeman, M. Benzaquen, T. Ondarçuhu, Dynamics of anchored oscillating nanomenisci, Physical Review Fluids 2 (10) (2017) 1-9. doi:10.1103/PhysRevFluids. 2.102201

[9] J. Gao, W. D. Luedtke, D. Gourdon, M. Ruths, J. N. Israelachvili, U. Landman, Frictional forces and Amontons' law: From the molecular to the macroscopic scale, Journal of Physical Chemistry B 108 (11) (2004) 3410-3425. doi:10.1021/jp0363621

[10] Y. H. Zhou, C. Y. Chen, B. L. Li, K. Q. Chen, Characteristics of classical Kirchhoff's superposition law in carbon atomic wires connected in parallel, Carbon 95 (2015) 503-510. doi:10.1016/j. carbon.2015.08.064.

[11] A. Khodayari, M. Fasano, M. Bozorg Bigdeli, S. Mohammadnejad, E. Chiavazzo, P. Asinari, Effect of interfacial thermal resistance and nanolayer on estimates of effective thermal conductivity of nanofluids, Case Studies in Thermal Engineering 12 (June) (2018) 454-461. doi:10.1016/j. csite.2018.06.005.

[12] S. Liu, X. F. Xu, R. G. Xie, G. Zhang, B. W. Li, Anomalous heat conduction and anomalous diffusion in low dimensional nanoscale systems, European Physical Journal B 85 (10).

[13] S. Lepri, R. Livi, A. Politi, Thermal conduction in classical low-dimensional lattices, Physics Reports 377 (1) (2003) 1-80. doi:10.1016/S0370-1573(02)00558-6 
[14] S. Maruyama, A molecular dynamics simulation of heat conduction of a finite length single-walled carbon nanotube, Microscale Thermophysical Engineering 7 (1) (2003) 41-50. doi:10.1080/ 10893950390150467 .

[15] N. P. Wasekar, S. M. Latha, M. Ramakrishna, D. S. Rao, G. Sundararajan, Pulsed electrodeposition and mechanical properties of Ni-W/SiC nano-composite coatings, Materials and Design 112 (2016) 140-150. doi:10.1016/j.matdes.2016.09.070.

[16] N. P. Wasekar, L. Bathini, G. Sundararajan, Tribological Behavior of Pulsed Electrodeposited Ni-W/SiC Nanocomposites, Journal of Materials Engineering and Performance 27 (10) (2018) 5236-5245. doi:10.1007/s11665-018-3608-z.

[17] Y. Zare, H. Garmabi, Analysis of Tensile Modulus of PP/Nanoclay/CaCO3 Ternary Nanocomposite Using Composite Theories, Journal of Applied Polymer Science 123 (4) (2012) 2309-2319. doi:10.1002/app.

[18] E. Esmizadeh, G. Naderi, M. H. R. Ghoreishy, Modification of Theoretical models to predict mechanical behavior of PVC/NBR/organoclay nanocomposites, Journal of Applied Polymer Science 130 (5) (2013) 3229-3239. doi:10.1002/app.39556.

[19] A. Khodayari, A. W. Van Vuure, U. Hirn, D. Seveno, Tensile behaviour of dislocated/crystalline cellulose fibrils at the nano scale, Carbohydrate Polymers 235 (October 2019) (2020) 115946. doi:10.1016/j.carbpol.2020.115946.

[20] Y. Habibi, L. A. Lucia, O. J. Rojas, Cellulose Nanocrystals : Chemistry, Self-Assembly , and Applications, Chemical Reviews 110 (2010) 3479-3500.

[21] Y. van der Spoel, E. Lindahl, B. Hess, G. Groenho, A. E. Mark, H. J. C. Berendsen, GROMACS: Fast, Flexible and Free., Journal of computer chemistry 19(6) (6) (2005) 1701-1718.

[22] K. N. Kirschner, A. B. Yongye, S. M. Tschampel, J. González-Outeiriño, C. R. Daniels, B. L. Foley, R. J. Woods, GLYCAM06: A Generalizable Biomolecular Force Field. Carbohydrates, Journal of computational chemistry 29 (2008) 622-655.

[23] A. W. Sousa Da Silva, W. F. Vranken, ACPYPE - AnteChamber PYthon Parser interfacE, BMC Research Notes 5(367). 
[24] D. A. Case, I. Y. Ben-Shalom, S. R. Brozell, D. S. Cerutti, T. E. Cheatham, V. W. D. Cruzerio, T. A. Darden, R. E. Duke, D. Ghoreishi, M. K. Gilson, H. Gohlke, A. W. Goetz, D. Greene, R. Harris, N. Homeyer, S. Izadi, A. Kovalenko, T. Kurtzman, T. S. Lee, S. LeGrand, P. Li, C. Lin, J. Liu, T. Luchko, R. Luo, D. J. Mermelstein, K. M. Merz, Y. Miao, G. Monard, C. Nguyen, H. Nguyen, i. Omelyan, A. Onufreiv, F. pan, R. Qi, D. R. Roe, A. Roitberg, C. sagui, S. SchottVerdugo, J. Shen, C. L. Simmerling, J. Smith, R. Salomon-Ferrer, J. Swails, R. C. Walker, J. Wang, H. Wei, R. M. Wolf, X. Wu, L. Xiao, D. M. York, P. A. Kollman, AMBER 2018, University of California, San Francisco.

[25] M. Parinello, A. Rahman, Polymorphic transitions in single crystals: A new molecular dynamics method, Journal of Applied Physics 52 (1981) 7182-7190.

[26] D. S. BIOVIA, Materials studio 2017 r2, San Diego: Dassault Systèmes.

[27] Y. Nishiyama, P. Langan, H. Chanzy, Crystal structure and hydrogen-bonding system in cellulose I $\beta$ from synchrotron X-ray and neutron fiber diffraction, Journal of the American Chemical Society 124 (31) (2002) 9074-9082. 